\title{
Testing for cystic fibrosis using allelic association
}

\author{
A J IVINSON*, A P READ*, R HARRIS*, M SUPER $\dagger$, M SCHWARZ $\dagger$, \\ J CLAYTON SMITH*, AND R ELLES* \\ From *the Department of Medical Genetics, St Mary's Hospital, Manchester M13 0JH; and †Clinical Genetics \\ Unit, Royal Manchester Children's Hospital, Pendlebury, Manchester M27 1HA.
}

\begin{abstract}
SUMmARY A particular haplotype defined by probes XV2c, KM19, and CS.7 at the D7S23 locus was found on $90 \%$ of chromosomes which carry cystic fibrosis (CF), but on only $11 \%$ of normal chromosomes in a UK sample of CF carriers. We show how such data can be used to calculate carrier risks for people with and without a family history of CF, and give examples of clinical applications. For parents or sibs of dead CF patients, phase and genotypes can often be assigned with only 1 to $2 \%$ error. However, this method is not suitable for prenatal testing where there is no history of CF; for couples with no family history, no fetus can be shown to be at more than $2 \%$ risk of being affected.
\end{abstract}

The first prenatal diagnoses of cystic fibrosis $(\mathrm{CF})$ used the microvillar enzyme test of Brock et al. ${ }^{1}$ This test can be applied to any pregnancy by amniocentesis at about 18 weeks of gestation, but its specificity is low unless the prior risk is 1 in 4 or greater. The discovery of DNA polymorphisms linked to the CF locus $^{2-4}$ allowed prenatal diagnosis to be done earlier in pregnancy using chorion villus biopsy, with a lower error rate. We have described the principle of using these DNA markers elsewhere ${ }^{5}$ and summarised our experience with our first 30 cases. $^{6}$ The main limitation of this test is that it is applicable only to a minority of families, that is, those who have a surviving affected child.

DNA amplification using the polymerase chain reaction (PCR) ${ }^{7}$ has extended the scope of gene tracking. It is now possible to use stored Guthrie blood spot cards ${ }^{8}$ or histological specimens ${ }^{9}$ to obtain DNA from a long dead child and to score polymorphisms more easily and rapidly than previously. Nevertheless, gene tracking still requires a family history of the disease and material from an affected patient.

The original DNA markers met and $\mathrm{pJ} 3 \cdot 11^{2-4}$ show less than $1 \%$ recombination with the $C F$ locus, ${ }^{10}$ but there is little allelic association with $\mathrm{CF}$ (that is, normal and CF chromosomes carry much the same distribution of marker types). A second generation of probes, XV2c, KM19, and CS.7, ${ }^{11} 12$ defines the locus $D 7 S 23$ which lies much closer to the CF locus. The clinical importance of these new markers lies not so much in the reduced recombina-

Received for publication 5 December 1988.

Revised version accepted for publication 13 January 1989 tion error compared to the earlier markers, but in the strong population association between particular D7S23 haplotypes and the CF disease allele.

This allelic association presumably exists because most of the CF disease genes in the European population are descended from a single ancestral mutation, and so CF chromosomes still tend to carry a particular D7S23 haplotype. By good fortune, the original mutation evidently happened on a chromosome carrying an unusual haplotype. As a result, most CF chromosomes in the UK carry a different D7S23 haplotype from most normal chromosomes. Thus, it now becomes possible to identify putative CF carrying chromosomes even in families where there is no material from an affected patient. We report here our experience of using these allelic associations to extend the scope of prenatal diagnosis and carrier detection, and show a simple way of calculating risks for any haplotype or genotype.

\section{Materials and methods}

We analysed 114 informative Caucasian families, mainly from the north western region of England. The polymorphisms studied are shown in table 1 . Conventional Southern blotting ${ }^{13}$ was used for the XV2c and some KM19 analyses. Some KM19 and all CS.7 analyses were done by polymerase chain reaction, ${ }^{7}$ using oligonucleotide primer sequences kindly communicated by Professor Bob Williamson. Starting with less than $1 \mu \mathrm{g}$ DNA, 30 rounds of amplification were carried out using a Peerless Systems programmable mechanical arm. One-third of each sample was removed, digested with the 
TABLE 1 RFLPs used and allele definitions.

\begin{tabular}{|c|c|c|c|c|c|c|c|}
\hline \multicolumn{4}{|c|}{ Conventional analysis } & \multicolumn{4}{|c|}{ PCR analysis } \\
\hline Probe & Enzyme & Allele & Size (kbp) & Marker & Enzyme & Allele & Size $(b p)$ \\
\hline $\mathrm{XV} 2 \mathrm{c}$ & TaqI & $\begin{array}{l}\mathrm{X} 1 \\
\mathrm{X} 2\end{array}$ & $\begin{array}{l}2.1 \\
1.4\end{array}$ & CS.7 & HhaI & $\begin{array}{l}\text { CS1 } \\
\text { CS2 }\end{array}$ & $\begin{array}{l}330 \\
165\end{array}$ \\
\hline
\end{tabular}

appropriate enzyme, and electrophoresed on $2 \%$ Nusieve agarose gels. RFLP types were read by UV fluorescence from gels stained with ethidium bromide (fig 1).

\section{RISK CALCULATIONS}

All risk estimates are based on simply counting numbers of $\mathrm{CF}$ and non-CF (NCF) chromosomes with each D7S23 haplotype and applying Bayes's theorem ${ }^{14}$ to the numbers.

Let $\mathrm{p}$ be the prior risk that a chromosome carries CF ( 1 in 2 for the sib of a patient and 1 in 45 for a randomly selected person in the UK). If haplotype $A$ is seen in c out of C CF chromosomes and n out of N NCF chromosomes, then the risk that an unknown chromosome with this haplotype carries CF is

$$
\mathrm{R}_{\mathrm{A}}=(\mathrm{p} \cdot \mathrm{c} / \mathrm{C}) /[\mathrm{p} \cdot \mathrm{c} / \mathrm{C}+(1-\mathrm{p}) \cdot \mathrm{n} / \mathrm{N}] \text {. }
$$

If a phenotypically normal person has haplotypes $A$ and $B$ with risk $R_{A}$ and $R_{B}$, the risk that he is a CF carrier is

$$
\left[R_{A}\left(1-R_{B}\right)+R_{B}\left(1-R_{A}\right)\right] /\left(1-R_{A} R_{B}\right) \text {. }
$$

A small BASIC program, available on request, has been written to do these sums. From standard statistics, there is a $95 \%$ chance that the true value of $\mathrm{c} / \mathrm{C}$ in the population lies within $1.96 \mathrm{SD}$ of the value observed in our sample, and the $\mathrm{SD}$ is $\sqrt{[c(C-c)] / C}$. Substituting $c / C(1.96 \mathrm{SD})$ in the calculation of carrier risk gave the confidence intervals shown in table 2.

\section{Results}

Table 3 shows the distribution of marker alleles and table 4 the distribution of haplotypes among normal (NCF) and CF chromosomes. These results are generally similar to data on XV2c-KM19-CS.7 haplotypes reported ${ }^{12}$ from the UK, Denmark, Spain, and Finland, and to XV2c-KM19 haplotypes from Holland ${ }^{15}$ and Germany. ${ }^{16}$ The distribution of haplotypes among both normal and CF chromosomes was not significantly different in our results and in the UK series of Estivill et al, ${ }^{12}$ and therefore these two series were combined for calculating risks. Tables 2 and 5 show the risks for each haplotype and genotype calculated by the method described. Inspection of table 2 shows that haplotype $D$ is associated with a high risk of carrying CF, and haplotypes $\mathrm{A}, \mathrm{B}, \mathrm{E}$, and $\mathrm{H}$ with a low risk.

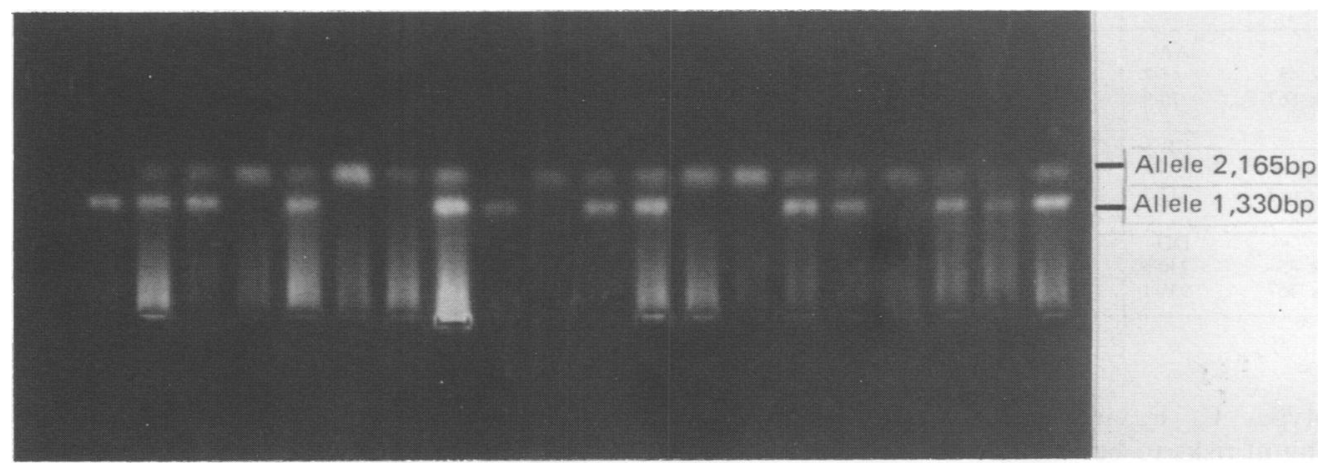

FIG 1 CS.7/Hhal polymorphism scored by polymerase chain reaction. 1 ug samples of genomic DNA were amplified for 30 cycles, digested with HhaI, and electrophoresed in a $2 \%$ Nusieve agarose gel. The fragments were visualised by UV fluorescence after staining with ethidium bromide. When the Hhal site is present the 330 bp amplified sequence is cleaved into two 165 bp fragments. 
TABLE 2 Risk that a chromosome of each common haplotype carries the CF gene, calculated using the combined data in table 4.

\begin{tabular}{lrrllc}
\hline Haplotype & \multicolumn{2}{c}{ Combined } & Nos & & \multicolumn{2}{l}{$1000 \times$ risk $\pm 95 \%$ CI } \\
\cline { 2 - 3 } \cline { 5 - 6 } \cline { 5 - 6 } & $C F$ & $N C F$ & & Prior $=1 / 2$ & Prior $=1 / 45$ \\
\hline A X1,K1,CS1 & 1 & 24 & & $36 \cdot 7 \pm 6$ & $0 \cdot 9 \pm 0 \cdot 1$ \\
B X1,K1,CS2 & 1 & 11 & & $76 \cdot 8 \pm 12$ & $1 \cdot 9 \pm 0 \cdot 3$ \\
D X1,K2,CS2 & 118 & 15 & & $878 \pm 5$ & $140 \pm 5$ \\
E X2,K1,CS1 & 7 & 51 & & $112 \pm 6$ & $2 \cdot 8 \pm 0 \cdot 2$ \\
H X2,K2,CS2 & 3 & 15 & & $155 \pm 12$ & $4 \cdot 1 \pm 0 \cdot 1$ \\
Others (C,F,G) & 0 & 3 & & - & - \\
\hline
\end{tabular}

TABLE 3 Distribution of alleles at the D7S23 locus in normal (NCF) and CF chromosomes in parents of $C F$ children.

\begin{tabular}{lrrrrrr}
\hline Allele & $X 1$ & $X 2$ & $K 1$ & \multicolumn{1}{c}{$K 2$} & \multicolumn{1}{c}{ CS1 } & CS2 \\
\hline CF chromosomes & 205 & 23 & 19 & 200 & 8 & 75 \\
NCF chromosomes & 94 & 132 & 164 & 55 & 50 & 33 \\
\hline
\end{tabular}

TABLE 4 D7S23 haplotypes in parents of CF children: our data compared to the data of Estivill et al. ${ }^{12}$ Not all chromosomes in our series were typed for CS.7.

\begin{tabular}{|c|c|c|c|c|c|c|}
\hline \multirow[t]{2}{*}{ Haplotype } & \multicolumn{2}{|c|}{$\begin{array}{l}\text { Estivill } \\
\text { et al }{ }^{12}(U K)\end{array}$} & \multicolumn{2}{|c|}{ Our data } & \multicolumn{2}{|c|}{$\begin{array}{l}\text { Our } X-K \\
\text { haplotypes }\end{array}$} \\
\hline & CF & NCF & CF & NCF & $\mathrm{CF}$ & NCF \\
\hline A X1,K1,CS1 & 0 & 12 & 1 & 12 & 3 & 49 \\
\hline B X1,K1,CS2 & 1 & 3 & 0 & 8 & & \\
\hline $\mathrm{C} \times 1, \mathrm{~K} 2, \mathrm{CS} 1$ & 0 & 1 & 0 & 0 & 147 & 19 \\
\hline D X1, K2, CS2 & 51 & 8 & 67 & 7 & & \\
\hline E X2,K1,CS1 & 5 & 24 & 2 & 27 & 8 & 70 \\
\hline F X2,K1,CS2 & 0 & 0 & 0 & 2 & & \\
\hline $\mathrm{G} \times 2, \mathrm{~K} 2, \mathrm{CS} 1$ & 0 & 0 & 0 & 0 & 8 & 25 \\
\hline $\mathrm{H} \times 2, \mathrm{~K} 2, \mathrm{CS} 2$ & 1 & 4 & 2 & 11 & & \\
\hline Total & 58 & 52 & 72 & 67 & 166 & 163 \\
\hline
\end{tabular}

TABLE 5 Risk $(\times 1000)$ that a phenotypically normal person with the given haplotypes (defined in table 4) is a CF carrier. Prior risks of 45 and 667 per thousand are the 1/22 population risk and the 2/3 risk for a sib of a CF patient, respectively.

\begin{tabular}{|c|c|c|c|c|c|}
\hline Genotype & $\mathbf{A A}$ & AB & AD & $\mathrm{AE}$ & $\mathrm{AH}$ \\
\hline Prior risk 45 & 1.7 & $2 \cdot 8$ & $141 \cdot 3$ & $3 \cdot 7$ & $5 \cdot 0$ \\
\hline \multirow[t]{2}{*}{ Prior risk 667} & $70 \cdot 9$ & $108 \cdot 2$ & $878 \cdot 6$ & $140 \cdot 7$ & $181 \cdot 1$ \\
\hline & BB & BD & $\mathrm{BE}$ & BH & $\mathbf{H H}$ \\
\hline Prior risk 45 & 3.8 & $142 \cdot 0$ & $4 \cdot 7$ & $6 \cdot 0$ & $8 \cdot 3$ \\
\hline \multirow[t]{2}{*}{ Prior risk 667} & $142 \cdot 7$ & $879 \cdot 3$ & $172 \cdot 8$ & $210 \cdot 3$ & $268 \cdot 0$ \\
\hline & DD & DE & DH & $\mathrm{EE}$ & EH \\
\hline Prior risk 45 & $246 \cdot 6$ & 142.7 & 143.7 & $5 \cdot 7$ & $7 \cdot 0$ \\
\hline Prior risk 667 & $935 \cdot 1$ & 879.9 & $880 \cdot 7$ & $200 \cdot 8$ & $235 \cdot 9$ \\
\hline
\end{tabular}

Haplotypes C, F, and $G$ are still too rare for meaningful risks to be calculated.

\section{Case reports}

The following cases illustrate the value of these associations in circumstances where gene tracking was not possible.

\section{CASE 1}

A 30 year old man reported a history of persistent sinusitis, malabsorption, azoospermia, and recurrent bronchopulmonary infection, with evidence of pulmonary tuberculosis and pancreatic steatorrhoea. . In childhood a diagnosis of CF had been overturned; however, the course of his illness suggested that $\mathrm{CF}$ was now a strong possibility. DNA analysis showed that he was homozygous for haplotype D. From table $2,82 \%$ of people with CF but only $1.6 \%$ of normal homozygotes (and $23 \%$ of CF carriers) are expected to have the DD genotype. The odds in favour of his having $\mathrm{CF}$, given these results, are

$$
0 \cdot 82:(0 \cdot 016+1 / 23 \times 0 \cdot 23)=31: 1 \text {. }
$$

Thus the DNA studies supported the diagnosis of $\mathrm{CF}$, and provided anecdotal support for the view that unusually mild CF may result from the same mutation as the classical disease.

\section{CASE 2 (FIG 2)}

A 29 year old nulliparous woman presented.at 11 weeks' gestation. Her sister had died many years before of CF, confirmed by necropsy. She and her non-consanguineous husband were given a risk for $\mathrm{CF}$ of $1 / 120$ in the present pregnancy, based on the assumptions that she had a $2 / 3$ and he a $1 / 20$ risk of being a carrier. The couple were unwilling to accept this $1 / 120$ risk and remained extremely anxious. DNA studies (fig 2) showed that both partners were homozygous for the low risk haplotype $\mathrm{E}$, while the woman's parents (both presumably carriers) each had one low risk (E) and one high risk (D) haplotype. The risk that the husband is a carrier, from table 5 , is $0.57 \%$. The wife's risk is lower than the figure of $20 \%$ in table 5 because of the results on her parents. For each parent, the phase could be

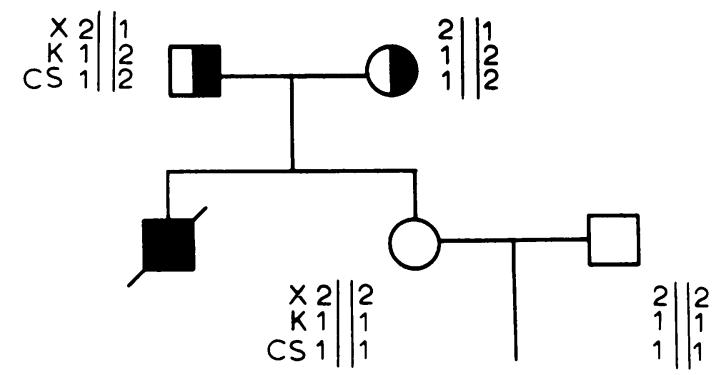

FIG 2 D7S23 haplotypes in case 2. $X=X V 2 c /$ TaqI, $K=K M 19 /$ Pst,$\quad C S=C S \cdot 7 /$ Hhal . 
$\mathrm{D}^{\mathrm{CF}} \mathrm{E}^{\mathrm{NCF}}$ or $\mathrm{D}^{\mathrm{NCF}} \mathrm{E}^{\mathrm{CF}}$ with equal prior probability. Each chromosome is at 1 in 2 prior risk of carrying $\mathrm{CF}$, therefore from table 2 the relative likelihoods of the two phases are

$$
0 \cdot 878 \times(1-0 \cdot 112):(1-0 \cdot 878) \times 0 \cdot 112=57: 1 .
$$

The chance that one or other of the woman's E haplotypes carries CF is $2 / 58$ or $3.5 \%$. Thus, the risk that both partners are carriers is $0.57 \% \times 3.5 \%$, and the risk of an affected child is 1 in 20000 .

\section{CASE 3 (FIG 3)}

A 41 year old woman presented at 10 weeks' gestation and gave a history of two CF children who died early in life followed by two healthy children. DNA analysis (fig 3) showed that the father was homozygous for haplotype $\mathrm{D}$, the mother was $\mathrm{BD}$, and the fetus was also BD. Its odds of inheriting a $\mathrm{CF}$ gene from the father were 1:1. Calculation of the mother's phase follows the method used in case 2 above. She could be $\mathrm{B}^{\mathrm{NCF}} \mathrm{D}^{\mathrm{CF}}$ or $\mathrm{B}^{\mathrm{CF}} \mathrm{D}^{\mathrm{NCF}}$ with equal prior probability, and each chromosome is at 1 in 2 prior risk. From table 2 the relative likelihoods of the two phases are

$$
(1-0.077) \times 0 \cdot 878: 0 \cdot 077 \times(1-0 \cdot 878)=86: 1 \text {. }
$$

Since the fetus inherited the B haplotype, it has a 1 in 87 chance of inheriting the CF gene from the mother, and an overall risk of 1 in 174 of being affected.

In this family we were able to confirm the low risk using $\mathrm{pJ} 3 \cdot 11$, one of the less closely linked markers. ${ }^{10}$ All five family members were heterozygous $2-1$. In isolation this result means that the two surviving unaffected boys must share either two or no haplotypes. Since one boy is $2-1$ and the other 2-2 with KM19 (fig 3), they cannot share two haplotypes, so they must share none. Given that neither boy is

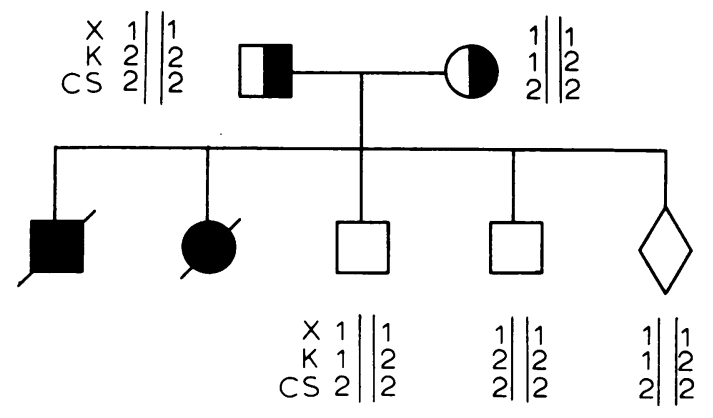

FIG 3 D7S23 haplotypes in case 3. X=XV2c/TaqI, $K=K M 19 / P s t I, C S=C S \cdot 7 / H h a I$. affected, they must both be carriers: one received CF from his father and NCF from his mother, the other vice versa. The fetus has the same genotype for all four markers as the older boy and therefore, barring recombination, it too is a carrier.

\section{Discussion}

We believe that the main clinical application of these results should be for prenatal diagnosis where couples are both known to be carriers but have no surviving affected child. The problem in these cases is to decide which chromosome in each parent carries CF; $90 \%$ of $\mathrm{CF}$ chromosomes carry haplotype D while $85 \%$ of NCF chromosomes carry haplotypes A, B, E, or $\mathrm{H}$. From the figures in table 2 we predict that over $75 \%$ of carriers will be $A D$, $\mathrm{BD}, \mathrm{ED}$, or $\mathrm{HD}$, and the chance of identifying the CF chromosome wrongly in such a carrier is only 1 to $1.5 \%$, as shown for cases 2 and 3 above. This compares with the $0.5 \%$ risk of error when the $\mathrm{CF}$ chromosome is identified by studying an affected child with a single marker from the met or pJ3.11 series. ${ }^{10} \mathrm{~A}$ second useful application is for carrier couples who have requested donor insemination. About half of all sperm donors carry only haplotypes $\mathrm{A}, \mathrm{B}$, or $\mathrm{E}$, and by using them the risk of a CF child is negligibly low.

For a person with no family history of $\mathrm{CF}$, carrying haplotype $D$ raises the carrier risk from $1 / 23$ to $1 / 7$. Even if the population were screened for couples both carrying haplotype $\mathrm{D}$, their DD fetuses would be at only $1 / 7 \times 1 / 7=2 \%$ risk of CF. Thus, there is no case for offering these tests to people without a family history of CF: population screening must await the definition of the CF mutation itself.

DNA testing is often requested by couples where one partner has no family history and the other has a dead CF sib, as in our case 2 . Those who persist with the request after counselling are presumably those who are unwilling to take a 1 in 120 risk (the risk before any DNA results). Some laboratories might refuse to do tests because of the likelihood that they will lead to terminations of pregnancies at relatively low risk. These problems cannot be entirely avoided, but they can be minimised by trying hard to establish haplotypes before a pregnancy, using other family members as necessary, and by haplotyping the high risk partner before doing any tests on the low risk partner. A possible protocol would be as follows.

(1) Establish haplotypes for the high risk partner. Unless this person has at least one D (high risk) haplotype, useful prenatal testing will not be possible. Given that he or she does not have a D haplotype, the next highest risk would be seen if the fetus inherits an $\mathrm{H}$ haplotype from the high 
risk and a $\mathrm{D}$ haplotype from the low risk parent. From table 2, the risk that the fetus is affected is $0 \cdot 155 \times 0 \cdot 14=2 \%$. This is too low for the microvillar enzyme test to be useful.

(2) If the high risk partner has at least one D haplotype, the haplotypes of the low risk partner are established next. In about half of all cases only the low risk haplotypes A, B, or E will be present. In these cases the couple can be reassured. Even if a fetus inherits haplotype D from the high risk partner, if it inherits $A, B$, or $\mathrm{E}$ from the low risk parent it is at no more than $0 \cdot 3 \%$ risk of being affected (table 2 ).

(3) A fetus which inherits haplotype D from both parents is at about $1 / 7$ risk, and perhaps the microvillar enzyme test would be appropriate later in the pregnancy.

All the results given here depend on being able to count the numbers of $\mathrm{CF}$ and non-CF chromosomes with each haplotype; hence, indeterminate haplotypes cause problems. These arise when the parents and the affected child are all heterozygous for two or more markers so that it is not possible to identify the $\mathrm{CF}$ and non-CF haplotypes. Sometimes analysis of grandparents or unaffected sibs will help, but some indeterminate cases will remain. As has been pointed out, ${ }^{16}$ the missing haplotypes are not a random selection of those in the population, and ignoring them could bias the estimates of haplotype frequencies. Only a minority of haplotypes are indeterminate (16/155 in our data), so any bias will not be serious for common haplotypes, but the frequency of some rare haplotypes might be underestimated. We used the Hapmax program ${ }^{16}$ (kindly made available by Dr $M$ Krawczak) to make a maximum likelihood estimate of the true haplotype frequencies from our data in table 4 . The only significant change was an increase in the frequency of haplotype $\mathrm{E}$ on $\mathrm{CF}$ chromosomes from $2 \cdot 8 \%$ $(2 / 72$, table 4$)$ to $6.9 \%$. The risks used throughout this paper are derived from combined data, ours plus those of Estivill et al, ${ }^{12}$ and in these combined data the $E$ frequency is $7 / 130=5 \cdot 4 \%$. We conclude that indeterminate haplotypes are not seriously biasing our risk estimates.

We thank Professor Bob Williamson and $\mathrm{Dr}$ Carolyn Williams for the probes XV2c, KM19, and CS.7 and the primer sequences used in the PCR, and Dr Dian Donnai for referring patients. This work was supported by the North Western Regional Health Authority, the Department of Health, and the Cystic Fibrosis Research Trust.

\section{References}

${ }^{1}$ Brock DJH, Bedgood D, Barron L, Hayward C. Prospective prenatal diagnosis of cystic fibrosis. Lancet 1985;i:1175-8.

2 Knowlton RG, Cohen-Hagenauer O, Nguyen VC, et al. A polymorphic DNA marker linked to cystic fibrosis is located on chromosome 7. Nature 1985;318:380-2.

3 White R, Woodward S, Leppert M, et al. A closely linked genetic marker for cystic fibrosis. Nature 1985;318:382-4.

4 Wainwright BJ, Scambler PJ, Schmidtke J, et al. Localisation of cystic fibrosis locus to human chromosome 7cen-7q22. Nature 1985;318:384-5.

5 Read AP. Principles of DNA-based prenatal diagnosis of cystic fibrosis: an introduction for the clinician. J R Soc Med 1987;80 (suppl 15):9-12.

6 Super M, Ivinson A, Schwarz M, et al. Clinical experience of prenatal diagnosis of cystic fibrosis by use of linked DNA probes. Lancet 1987;ii:782-4.

7 Saiki RK, Scharf S, Faloona F, et al. Enzymatic amplification of $\beta$-globin genomic sequences and restriction site analysis for diagnosis of sickle cell anemia. Science 1985;230:1350-4.

8 Williams C, Weber L, Williamson R, Hjelm M. Guthrie spots for DNA-based carrier testing in cystic fibrosis. Lancet 1988;ii: 693.

9 Imprain CC, Saiki RK, Ehrlich HA, Teplitz RL. Analysis of DNA extracted from formalin-fixed paraffin-embedded tissues by enzymatic amplification and hybridisation with sequencespecific oligonucleotides. Biochem Biophys Res Commun 1987 142:710-6.

10 Beaudet A, Bowcock M, Buchwald M, et al. Linkage of cystic fibrosis to two tightly linked DNA markers. Joint report from a collaborative study. Am J Hum Genet 1986;39:681-93.

11 Estivill X, Farrall M, Scambler PJ, et al. A candidate for the cystic fibrosis locus isolated by selection for methylation-free islands. Nature 1987;326:840-5.

12 Estivill X, Scambler PJ, Wainwright BJ, et al. Patterns of polymorphism and linkage disequilibrium for cystic fibrosis Genomics 1988;1:257-63.

${ }^{13}$ Maniatis T, Fritsch EF, Sambrook J, eds. Molecular cloning: a laboratory manual. New York: Cold Spring Harbor Laboratory, 1982.

14 Vogel F, Motulsky AG. Human genetics. Berlin: SpringerVerlag, 1979:594.

15 te Meerman GJ, ten Kate LP, Cobben JM, von Essen T, Buys CHCM, Halley D. Prenatal diagnosis of cystic fibrosis where single affected child has died. Lancet 1988;ii:745.

16 Krawczak M, Konecki D, Schmidtke J, et al. Allelic association of the cystic fibrosis locus and two DNA markers, XV2-c and KM19, in 55 German families. Hum Genet 1988;80:78-80.

Correspondence to Dr A P Read, Department of Medical Genetics, St Mary's Hospital, Hathersage Road, Manchester M13 0JH. 